\title{
Characterization of Degradation under Standard Environmental Testing Methods for Crystalline Silicon Photovoltaic Modules
}

\author{
Sagarika Kumar*, Subinoy Roy and Rajesh Gupta \\ Department of Energy Science \& Engineering, Indian Institute of Technology Bombay, Mumbai-400076, India \\ Email: sagarika91@iitb.ac.in
}

\begin{abstract}
Standard environmental tests have been developed for photovoltaic (PV) modules to assess the reliability of their performance in a short period of time. These tests generate different modes of degradation by combination of environmental stresses. There is a need to differentiate between the standard tests on the basis of severity. This helps to understand the individual and combined degradation effect occurred due to temperature and humidity stress, which is the focus of the present study. In this work, standard tests viz. humidity freeze (HF), thermal cycling (TC) and damp heat test $(\mathrm{DH})$ have been performed on different batches of multi-crystalline silicon PV modules. One batch of modules was subjected to combined tests wherein the three tests were performed in succession and the other batch was separately subjected to the individual tests. Spatial characterization techniques i.e. electroluminescence (EL) and dark lock-in-thermography (DLIT) imaging were used in tandem with illuminated current voltage (I-V) analysis for detection and quantification of degradation under the standard tests. The information obtained from the comparison between the different tests, on a scale of time and severity can be helpful for preliminary investigations performed on effects of the common environmental degradation factors like high temperature, high humidity and thermal ramping. The presented findings can further aid research and development on different aspects of reliability testing on PV module performance under environmental effects.
\end{abstract}

Keywords: Photovoltaic, multicrystalline silicon modules, reliability, degradation, defects, characterization

\section{Introduction}

The recent large scale deployment of multi crystalline silicon photovoltaic (PV) modules in the field has raised the need for in-depth studies of degradation in a fast and efficient manner. In this context, different standard test procedures as specified under IEC 61215 viz. thermal cycling (TC), humidity freeze $(\mathrm{HF})$ and damp heat $(\mathrm{DH})$ test have been developed for crystalline silicon PV modules [1]. These tests undergo different degradation mechanisms on application of various environmental stress factors in an accelerated frame and help in assessing the reliability of the modules in a short period of time [2-4]. These tests when performed on the PV modules, check for the modules reliability under different aspects such as high temperature effect, thermal ramping, and humidity ingression effect. These aspects essentially affect the module performance individually or in combination to one another. It has been reported that temperature variation causes breakage of interconnects, cells and solder joints [5-7]. The thermal fatigue essentially causes accumulation of strain energy due to the temperature loading, which causes such failures $[8,9]$. Whereas, moisture ingression through the back sheet or laminate is the mode of degradation at high humidity conditions [10,11]. Presence of humidity weakens the interfacial adhesive bonds that further result into increased ingress paths that expedite loss of passivation and corrosion [12-14].

The above-mentioned degradation factors essentially affect the module performance and arises the need for in-depth investigation. Various spatial and non-spatial techniques viz. illuminated current voltage (I-V) analysis, electroluminescence (EL) and dark lock-in-thermography (DLIT) have been 
capable in detection and analysis of defects in PV modules under the standard reliability tests. I-V analysis has been used for extraction of global electrical parameters in relation to the degradation observed under the tests [15]. However, I-V analysis alone cannot be used for defect analysis as it does not indicate the cause of the degradation. Hence, it is essential to obtain spatially resolved information of the PV modules to understand the channel of degradation. Hereof, EL and DLIT imaging in addition to non-spatial I-V investigation can be exploited for detection and analysis of defects in PV modules. These techniques also provide an advantage of being fast and non-destructive. In this regard, EL imaging technique is extensively used in the production line during the fabrication process of the solar cells to identify process induced defects like grain boundaries and inhomogeneity caused by dislocation. Also, mechanical damage to the wafer, fingers and other electrical contacts can be detected by this technique [7,16]. Whereas, DLIT technique has not been vastly used for PV module investigations as the interpretation of the results are not straight forward as compared to solar cell applications. It has dominantly been used for localization of electrical defects such as shunts [17-19]. Recently, the use of DLIT technique has been demonstrated for detection and characterization of module packaging defects like delamination and discoloration that cause degradation in the long term [20,21].

In context of degradation studies, earlier studies have been using I-V analysis and EL imaging for detection of commonly occurring defects under the individual standard tests. However, it can be helpful to perform a comparative assessment on the working nature of different tests. This will be instrumental in providing information on the common modes of degradation due to different combination of temperature and humidity stressors.

In this study, three standard qualification tests viz. TC, HF and DH are studied on multi-crystalline silicon PV modules followed by defect characterization by IV analysis, EL and DLIT imaging techniques. These tools have been used in tandem for the analysis of the observed degradation after the standard tests by extraction of the spatially resolved information of the defects and degradation in the modules. The test has been conducted on two module batches as shown in figure 2. Combined tests were conducted to observe the difference in mode of degradation when conducted in succession. The tests have been compared on the basis of the observed degradation in output power $\left(\mathrm{P}_{\max }\right)$ along with correlation with images obtained after the spatial characterization of the PV modules.

\section{$2 \quad$ Materials and Methods}

The study has been carried out on batches of multi-crystalline silicon PV modules from the same manufacturer. The samples have been chosen with similar electrical and geometrical parameters for a proper comparative study. All the modules had an area of $0.24 \mathrm{~m}^{2}$ with 36 cells in series. The electrical parameters obtained for the study are maximum output power $\left(\mathrm{P}_{\max }\right)$, series resistance $\left(\mathrm{R}_{\mathrm{s}}\right)$, short circuit current $\left(\mathrm{I}_{\mathrm{sc}}\right)$, fill factor $(\mathrm{FF})$ and open circuit voltage $\left(\mathrm{V}_{\mathrm{oc}}\right)$. The average initial values with their respective standard deviations from the mean value have been shown in table 1.

Table 1. Electrical characteristic parameters of sample PV modules.

\begin{tabular}{llllll}
\hline & $\mathbf{P}_{\max }(\mathbf{W})$ & $\mathbf{R}_{\mathrm{s}}(\boldsymbol{\Omega})$ & $\mathbf{I}_{\text {sc }}(\mathbf{A})$ & $\mathbf{F F}(\%)$ & $\mathbf{V}_{\text {oc }}(\mathbf{V})$ \\
\hline Mean Value & $28.36 \pm 0.28$ & $1.49 \pm 0.11$ & $1.6 \pm 0.02$ & $75 \pm 0.57$ & $23.5 \pm 0.15$ \\
\hline
\end{tabular}

Standard test procedures have been performed in an environmental chamber, which was dedicated to run the standard tests on PV modules. Thermal cycling (TC), humidity freeze (HF) and damp heat $(\mathrm{DH})$ have been performed according to the test conditions as specified under the IEC 61215 standard for an equal time duration of 250 hours for a time dependent comparative study. TC test consisting of a set of 50 cycles, of 5 hours each has been conducted at a temperature of $+85^{\circ} \mathrm{C}$ to $-40^{\circ} \mathrm{C}$ with a ramp rate of $1.67^{\circ} \mathrm{C} / \mathrm{min}$, as shown in figure 1 (a). HF test consisting of a sequence of 10 cycles, of 24 hours each has been carried out at a temperature of $+85^{\circ} \mathrm{C}$ to $-40^{\circ} \mathrm{C}$ at a similar ramp rate to TC along with 1 hour of dwell period at $85^{\circ} \mathrm{C} / 85 \% \mathrm{RH}$, as shown in figure $1(\mathrm{~b})$. DH test was performed at constant $85^{\circ} \mathrm{C} / 85 \% \mathrm{RH}$ for 250 hours. 


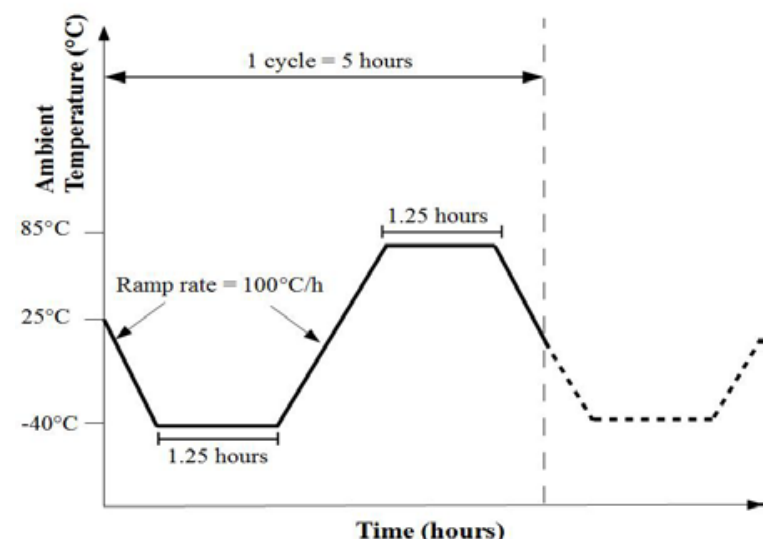

(a)

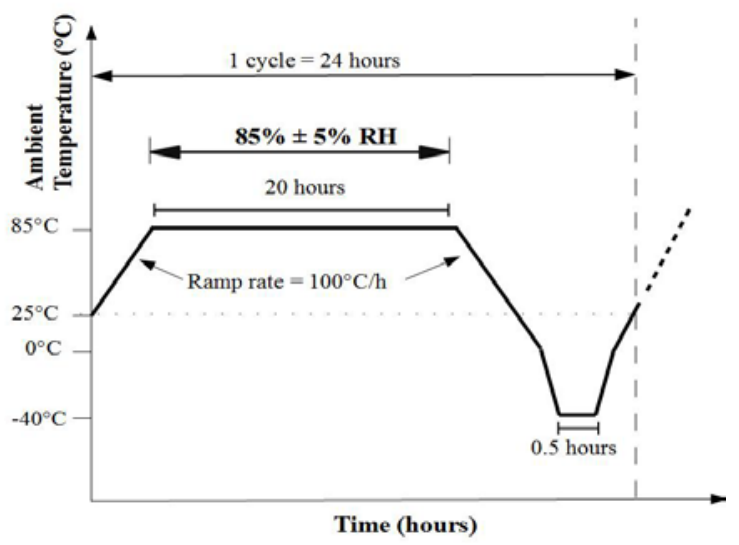

(b)

Figure 1. Test profile for (a) Thermal cycling and (b) Humidity Freeze test.

Sample modules have been divided in two batches I and II according to the different sequences of the standard test conditions. Batch-I modules have been subjected to the three tests in succession, in the order of HF, TC and DH for similar duration of operation to see the progressive effect of the sequence of tests on the PV modules. In batch-II different modules were subjected to the individual tests separately to compare between the individual degradation effects of the tests. The detailed test sequence has been described in figure 2. The module batches have been visually inspected and pre-characterized by IV, EL and DLIT techniques. These measurements were used as reference for the degradation study under the standard tests. Later, these techniques have been used after every step of the test procedure for investigation of defects.

Electroluminescence (EL) imaging is a rapid technique for characterization of defects, it detects and maps the luminescence emission of minority carriers in forward biased solar cell which emits radiation via radiative recombination of the minority carriers. The resultant EL emmision is in proportion to the total excess minority carrier density of the material [22]. The reduced minority carrier density regions can be spatially detected as lower intensity regions or dark spots, which can be easily visualised. The schematic of the experimental EL setup used is given in figure 3. The setup consists of silicon based charged couple device (CCD) camera, a programmable power supply and the mc-Si PV module. The programmable power supply works as an excitation source for PV modules, by forward biasing them at short circuit current value $\left(I_{\mathrm{sc}}\right)$. The $1024 \times 1024$ pixels silicon CCD acts as a detector of the luminescence emission emitted from the solar cells in the forward bias condition. The camera was actively cooled to reduce the thermal noise experienced by the detector. The position between the module and the camera was fixed in a dark enclosure to maintain spatial uniformity in the image measurements. The data acquisition time of 3 seconds was kept same for all the measurements. The experiment was performed in a dark room maintained at a constant temperature of $25^{\circ} \mathrm{C}$ to avoid interference with stray light.

Dark lock-in thermography (DLIT) imaging is a type of active thermography technique for investigation of PV modules under dark conditions, by passing electrical current through them. It detects the defects causing uneven power dissipation in the cells that results in temperature variation over the module surface. The imaging is performed from the thin back-sheet as it opaque to IR radiation in contrast to the glass in the front which is transparent to IR radiation. The schematic of the experimental DLIT setup used is similar to that of EL imaging technique given in figure 3, but the imaging is performed from the back-sheet of the PV module. The setup consists of an infrared (IR) camera, a programmable power supply, control unit and the mc-Si PV module. An 320 x 256 pixels cooled indium antimonide (InSb) detector IR camera in the spectral range of 3-5 $\mu \mathrm{m}$ has been used for the measurement at $150 \mathrm{~Hz}$ frame rate and $0.5 \mathrm{~Hz}$ frequency. The programmable power supply has been used as an excitation source by passing periodic electrical current in the module to create a thermal contrast at the back surface of the module. The total acquisition time for each module has been optimized at 120 seconds. The control unit synchronizes the power supply excitation with the frames of 
IR camera in order to implement lock-in algorithm on the captured images. The position between the module and the camera was fixed in a dark enclosure to maintain spatial uniformity in the images measurements. All the measurements were performed at constant temperature of $25^{\circ} \mathrm{C}$.

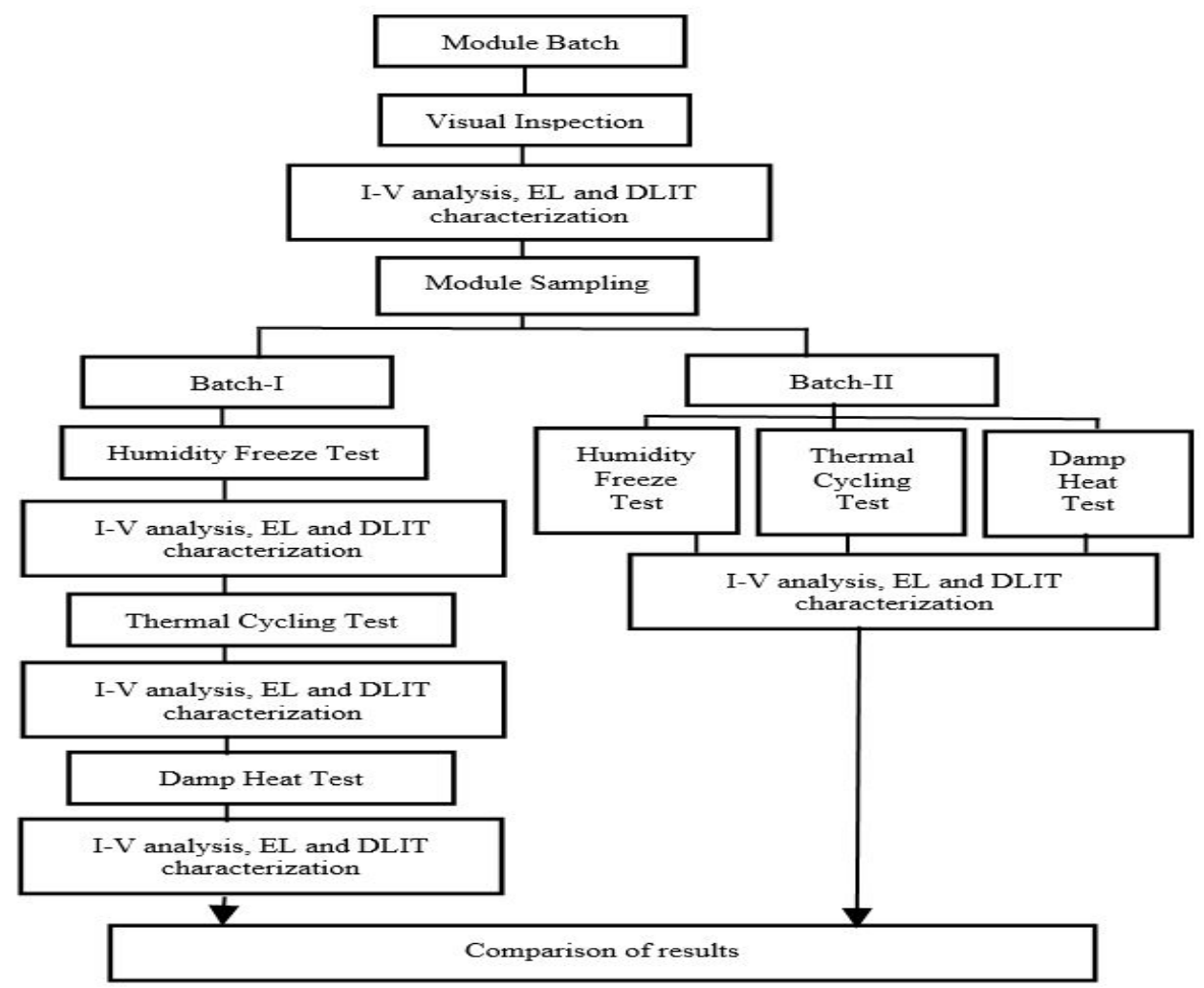

Figure 2. Experimental methodology.

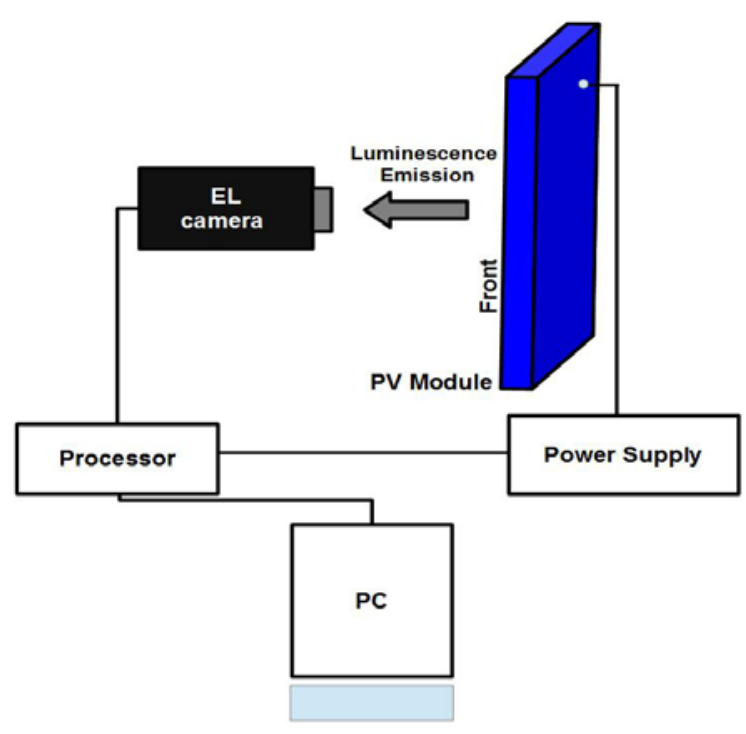

Figure 3. Schematic of the EL imaging setup for PV modules. 


\section{$3 \quad$ Results}

In this section, the experimental results from EL and DLIT imaging techniques are discussed for analysis of degradation observed under the standard reliability tests for batch-I and II modules. I-V analysis has been presented to investigate the preliminary signs of degradation and has been correlated with the results obtained from the spatial images.

\subsection{Illuminated Current Voltage (I-V) Analysis}

TC, HF and DH have been performed for an equal time duration of 250 hours on both the batches of PV modules. Visual inspection of the PV modules did not reveal any defects visible to the naked eye after the tests. The loss in the electrical parameters obtained from the I-V analysis after each test has been shown in table 2 for both the batches. I-V analysis shows significant degradation in maximum output power $\left(\mathrm{P}_{\max }\right)$ for all the modules. Here, $\mathrm{P}_{\max }$ has been observed to degrade in the decreasing order of $\mathrm{TC}, \mathrm{HF}$ and $\mathrm{DH}$ for the batch-II modules where modules were subjected to the individual tests separately. The variation in $I_{s c}$ and $V_{o c}$ is observed to be non-significant $(<1 \%)$ for the duration of the test. Loss in fill factor $(\mathrm{FF})$ has been observed as a major contributor for the loss in output power. One to one correspondence has been observed between the values of $\mathrm{FF}$ and $\mathrm{P}_{\max }$. The loss in FF loss has been attributed to the increment in series resistance $\left(R_{s}\right)$ in the observed modules. The maximum degradation in $\mathrm{P}_{\max }$ has been observed after TC $(\sim 7 \%)$ where the $\mathrm{R}_{\mathrm{s}}$ increased the most $(\sim 20 \%)$, on the other hand the loss in power after $\mathrm{DH}$ is observed to be about $2 \%$ with an increment in $\mathrm{R}_{\mathrm{s}}$ of almost $5 \%$. The I-V analysis of the batch-I modules also showed similar results where $\mathrm{P}_{\max }$ was observed to degrade in the decreasing order of TC, $\mathrm{HF}$ and $\mathrm{DH}$ and the reason for loss in $\mathrm{P}_{\max }$ was also loss in FF. In order to validate the effect of $\mathrm{TC}$, an additional 10 cycles of $\mathrm{HF}$ were performed on the batch-II HF test modules. Here, the degradation in $\mathrm{P}_{\max }$ was $5 \%$, whereas, the difference in $\mathrm{P}_{\max }$ from batch-I TC modules was $7 \%$.

Table 2. Loss in electrical parameters under the test on batch I and batch II module.

\begin{tabular}{lllllll}
\hline \multirow{2}{*}{ Degradation in parameters (\%) } & \multicolumn{3}{l}{ Batch-I } & \multicolumn{3}{c}{ Batch -II } \\
\cline { 2 - 7 } & HF & TC & DH & HF & TC & DH \\
\hline $\mathrm{P}_{\max }$ & 4.3 & 7.1 & 2.8 & 4.6 & 6.7 & 1.7 \\
$\mathrm{FF}$ & 3.9 & 7.0 & 2.2 & 4.5 & 6.5 & 1.3 \\
$\mathrm{R}_{\mathrm{s}}$ & 16.3 & 20.5 & 5.0 & 16.0 & 20.1 & 5.1 \\
\hline
\end{tabular}

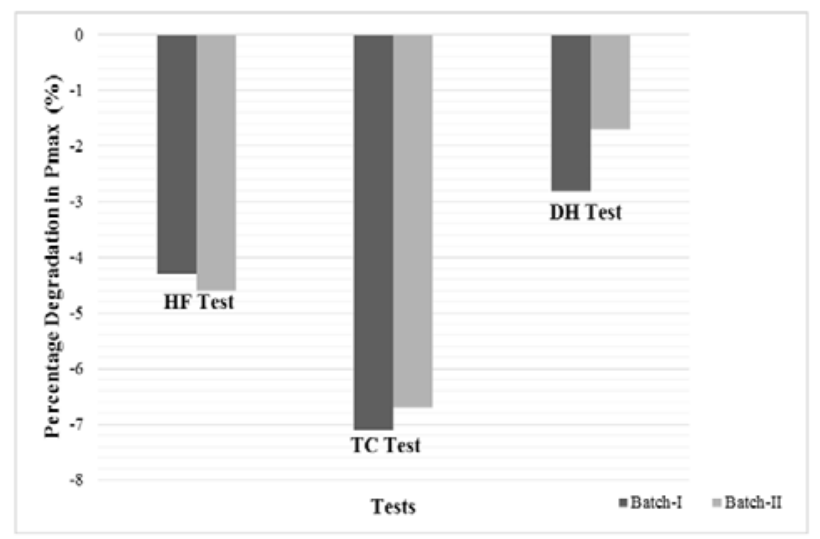

Figure 4. Comparison of power degradation under tests on batch-I and batch-II modules.

On comparison of the results between the two test batches, larger degradation in $\mathrm{P}_{\max }$ was observed for batch-I modules as compared to batch-II modules, as shown in figure 4 . To establish the difference 
between these two test batches, the change in $\mathrm{P}_{\max }$ for $\mathrm{DH}$ which was performed last in the combined test series can be observed. Here, for the two batches of $\mathrm{DH}$ the degradation in $\mathrm{P}_{\max }$ was $2.8 \%$ and $1.7 \%$ for batch I and II respectively. This shows that some extra effect of the preceding tests HF and TC was observed on the DH test modules, which was last in the sequence.

\subsection{Spatial Characterization of the Degraded Modules}

EL and DLIT imaging was carried out to extract the spatially resolved information about the defects obtained in the module batches, and also for establishment of correspondence with the results obtained from the I-V analysis. In the EL images of both the batches, distinct rectangular shaped areas were observed after both HF and TC tests as shown in figure 5(a)(i)-(iii). These can be identified as broken front grid fingers. On comparison of these images with the I-V results, it can be inferred that these broken fingers have caused an increment in $R_{s}$. Such distinct dark areas did not appear after the DH tests for the modules as visible in figure 5(a)(iv) and 7(a)(ii). On observing the progression of the finger defects, after TC test in the batch-I modules, the number of finger defects was observed to increase in some cell portions that appeared completely dark as shown in figure 5(a)(iii). The completely dark areas can be attributed to the absolute detachment of fingers from the wafer. These regions do not participate in the current carrying process, and cause the degradation in $\mathrm{P}_{\max }$. These regions are also visible as dark areas in the DLIT images as current flowing through the busbar into these regions is negligible, as seen in figure 5(b)(ii)-(iii) and 6(b)(ii)-(iii). It was also observed that the trend of number of finger defects corresponds to the increase in $\mathrm{R}_{\mathrm{s}}$ after all the tests. Some bright spots were observed in the DLIT images initially before the tests. These can be identified as localized shunts present in the module during the manufacturing or fabrication process.

(a)

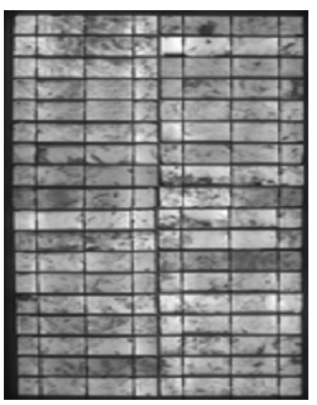

(i)

(b)

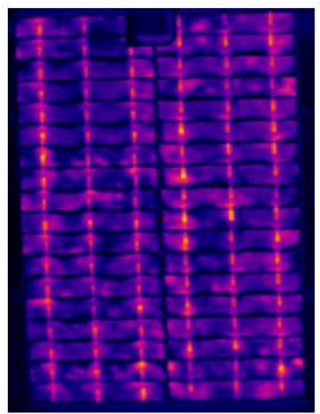

(i)

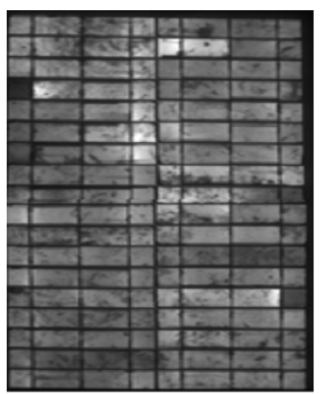

(ii)

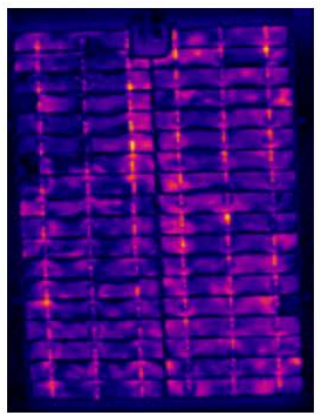

(ii)

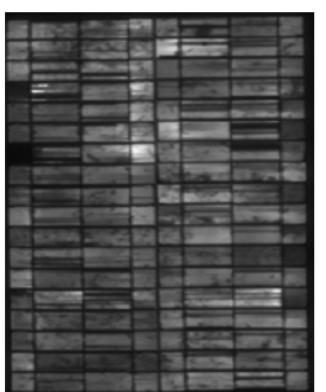

(iii)

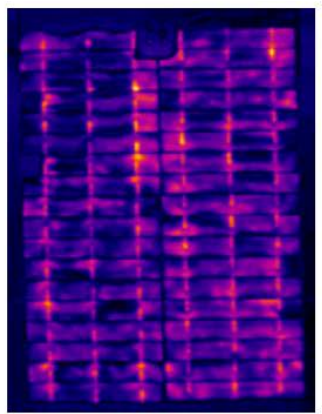

(iii)

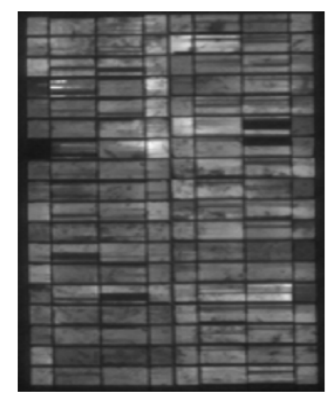

(iv)

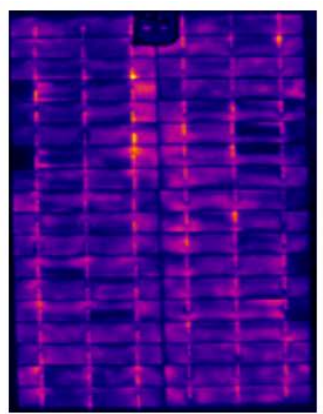

(iv)

Figure 5. (a) EL images of batch I modules (i) initially (ii) after 10 cycles of HF (iii) after 50 cycles of TC (iv) after 250h of DH; (b) DLIT images of batch I modules (i) initially (ii) after 10 cycles of HF (iii) after 50 cycles of TC (iv) after $250 \mathrm{~h}$ of $\mathrm{DH}$. 
(a)

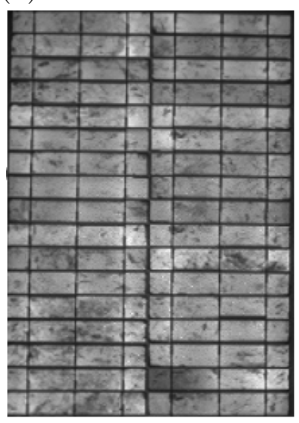

(i)

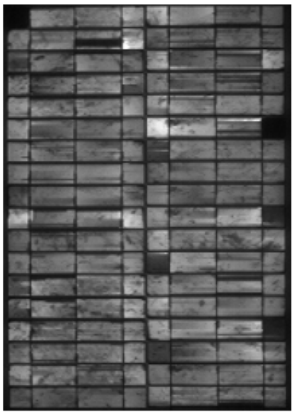

(ii) (b)

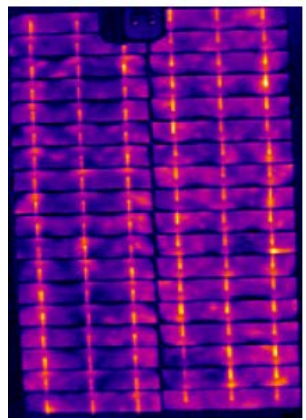

(i)

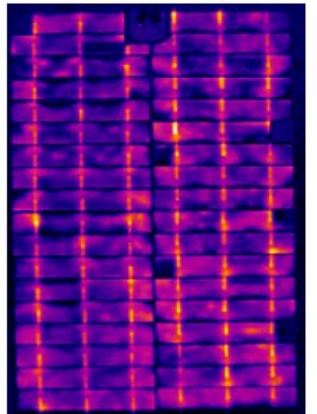

(ii)

Figure 6. (a) EL images of batch II modules (i) initially (ii) after 50 cycles of TC; (b) DLIT images of batch II modules (i) initially (ii) after 50 cycles of TC.

(a)

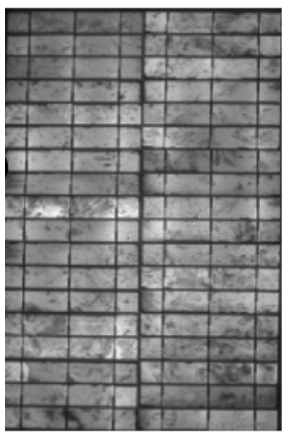

(i)

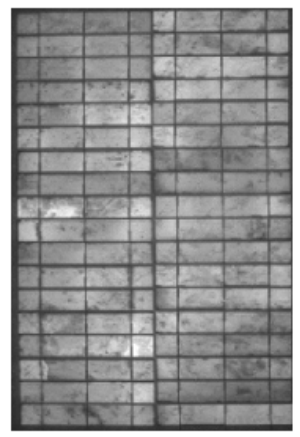

(ii)

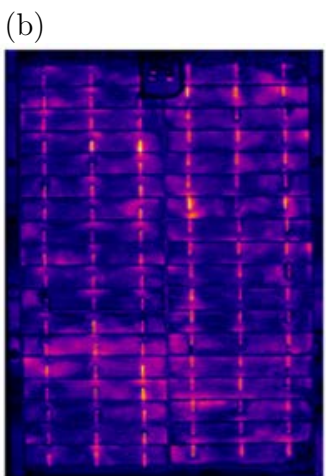

(i)

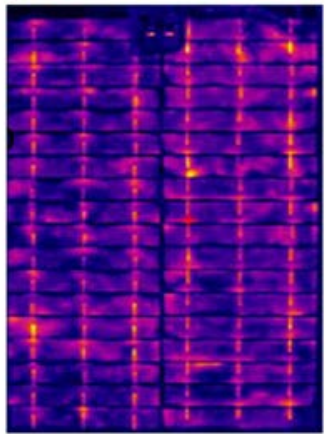

(ii)

Figure 7. (a) EL images of batch II modules (i) initially (ii) after $250 \mathrm{~h}$ of DH; (b) DLIT images of batch II modules (i) initially (ii) after $250 \mathrm{~h}$ of $\mathrm{DH}$

\section{Discussions}

The comparison of the amount of degradation between the three tests indicates that HF and TC test cause an earlier attainment of degradation in comparison to $\mathrm{DH}$ test. This can be due to the cyclic nature of $\mathrm{TC}$ and $\mathrm{HF}$ tests where the ramping between the high and low temperature accelerates the process of degradation. In contrast, the constant high temperature and humidity in case of DH test operates on moisture ingression which is a long term mode of degradation. This essentially highlights the severity of the thermo-mechanical fatigue over the moisture ingression mode of degradation. Further, comparison of TC and HF test shows faster and higher degradation in the case of TC test. The dominant portion of the $\mathrm{HF}$ test consists of a dwell period of constant temperature and humidity (Figure 1(b)), it is likely that this portion of the HF test cycle delays the degradation in comparison to TC test cycle.

The comparison of the two module batches shows extra amount of degradation in case of DH modules in batch-I in comparison to batch-II modules (Table 2). This can be termed as lingering effect of the degradation due to preceding tests on the subsequent one. It can be due to the effect of accumulated degradation due to earlier tests in sequence, which accelerates the moisture ingression process in DH test causing earlier degradation for batch-I DH modules. The obtained results signify the importance of investigations based on the environmental degradation factors viz. constant high temperature and humidity and thermal ramping on the degradations observed in the PV modules on scale of time and severity. 


\section{Conclusions}

In this paper, qualitative estimation and analysis of degradation after standard tests viz. thermal cycling, humidity freeze and damp heat test has been presented by dark lock-in-thermography and electroluminescence imaging on multi-crystalline silicon PV modules. Illuminated I-V characteristic parameters were used in support of the spatial images for quantification of degradation in terms of electrical parameters after the standard tests. Results were compared on a scale of time and severity in terms of loss in output power. Cyclic tests were found to cause more degradation in comparison to the non-cyclic test. Finger defects were found to be the major cause of degradation after the cyclic tests, visible as dark rectangular regions in the EL images. Corresponding loss in power was observed from the $\mathrm{I}-\mathrm{V}$ analysis dominantly due to loss in fill factor attributed to increment in series resistance. TC test was found to be the most severe causing maximum degradation in comparison to DH test in a fixed duration of time. A lingering effect of TC and HF test was observed on DH test which showed the dependence of the tests in a sequence. The interpretations from both the spatial and non-spatial characterization techniques led to similar findings and were found to be in correspondence to each other. The information supported for comparison of different tests can be insightful for the preliminary investigations of the commonly observed degradations affecting the PV modules due to the different combinations of temperature and humidity stressors. It can further be instrumental in designing of tests suitable for testing of PV modules in different conditions on basis of environmental degradation factors like high temperature, rapid cycling and high humidity.

\section{References}

1. IEC 61215, crystalline silicon terrestrial photovoltaic (PV) modules - Design qualification and type approval, 2005.

2. L.N. Dumas and A. Shumka, "Photovoltaic module reliability improvement through application testing and failure analysis", IEEE Transactions on Reliability, vol. R-31, pp. 228-234, 1982.

3. C. Ferrara and D. Philipp, "Why do PV modules fail?", in International conference on materials for advanced technologies, 2011. Proceedings. Energy Procedia, 2011.

4. A. Ndiaye, A. Charki, A. Kobi, C. M. F. Kébé, P. Ndiaye, and V. Sambou, "Degradations of silicon photovoltaic modules: A literature review," Solar Energy, vol. 96, pp. 140-151, 2013.

5. S. Kajari-Schröder, I. Kunze, and M. Köntges, "Criticality of cracks in PV modules", in silicon PV conference, 2012. Proceedings. Energy Procedia, vol. 27, pp. 658-663, 2012.

6. C. Oh, A. Kim, J. Kim, J. Bang, J. Ha, and W.S. Hong, "Bonding copper ribbons on crystalline photovoltaic modules using various lead-free solders," Journal of Materials Science: Materials in Electronics, vol. 26, no. 12, pp. 9721-9726, 2015.

7. P. Chaturvedi, B. Hoex and T.M. Walsh, "Broken metal fingers in silicon wafer solar cells and PV modules", Solar Energy Materials and Solar Cells, vol. 108, pp. 78-81, 2013.

8. M. T. Zarmai, N. N. Ekere, C. F. Oduoza, and E. H. Amalu, "Optimization of thermo-mechanical reliability of solder joints in crystalline silicon solar cell assembly," International Journal of Mechanical Engineering, vol. 59, pp. 117-125, 2016.

9. N. S. Bosco, T. J. Silverman, and S. R. Kurtz, "On the effect of ramp rate in damage accumulation of the CPV die-attach," in IEEE Photovoltaic Specialists Conference, 2012.

10. M. A. Munoz, M. C. Alonso-García, N. Vela, and F. Chenlo, "Early degradation of silicon PV modules and guaranty conditions," Solar Energy, vol. 85, no. 9, pp. 2264-2274, 2011.

11. W. Oh, S. Kim, S. Bae, N. Park, Y. Kang, H.S. Lee, and D. Kim, "The degradation of multi-crystalline silicon solar cells after damp heat tests," Microelectronics Reliability, vol. 54, pp. 2176-2179, 2014.

12. N. Park, C. Han, and D. Kim, "Effect of moisture condensation on long-term reliability of crystalline silicon photovoltaic modules," Microelectronics Reliability, vol. 53, no. 12, pp. 1922-1926, 2013.

13. K. R. McIntosh and X. Dai, "Damp-heat degradation and repair of oxide-passivated silicon," Physica Status Solidi (A) Applications and Material Sciences, vol. 208, no. 8, pp. 1931-1936, 2011. 
14. C. Peike, S. Hoffmann, P. Hülsmann, B. Thaidigsmann, K. A. Weiß, M. Koehl, and P. Bentz, "Origin of dampheat induced cell degradation," Solar Energy Materials and Solar Cells, vol. 116, pp. 49-54, 2013.

15. R. Khatri, S. Agarwal, I. Saha, S. K. Singh, and B. Kumar, "Study on long term reliability of photo-voltaic modules and analysis of power degradation using accelerated aging tests and electroluminescence technique", in Silicon PV, 2012. Proceedings. Energy Procedia, vol. 8, pp. 396-401, 2011.

16. T. Fuyuki and A. Kitiyanan, "Photographic diagnosis of crystalline silicon solar cells utilizing electroluminescence," Applied Physics A: Materials Science and Processing, vol. 96, no. 1, pp. 189-196, 2009.

17. P. Somasundaran, A. Sinha and R. Gupta, "Simulation and characterization of spatial variation of shunts in industrial solar cells by PSPICE and Dark Lock-in Thermography", in 27th European Photovoltaic Solar Energy Conference and Exhibition, 2012.

18. P. Somasundaran and R. Gupta, "Evaluation of shunt losses in industrial silicon solar cells," International Journal of Photoenergy, vol. 2016, 2016.

19. R. Gupta, P. Somasundaran, and D. K. Nandi, "Electrical simulation and characterization of shunts in solar cells," Applied Mechanics and Materials, vol. 110-116, no. 1, pp. 2453-2457, 2011.

20. A. Sinha, O. S. Sastry, and R. Gupta, "Nondestructive characterization of encapsulant discoloration effects in crystalline-silicon PV modules," Solar Energy Materials and Solar Cells, vol. 155, pp. 234-242, 2016.

21. A. Sinha, O. S. Sastry, and R. Gupta, "Detection and characterisation of delamination in PV modules by active infrared thermography," Nondestructive Testing and Evaluation, vol. 31, no. 1, pp. 1-16, 2016.

22. T. Fuyuki, H. Kondo, Y. Kaji, A. Ogane, and Y. Takahashi, "Analytic findings in the electroluminescence characterization of crystalline silicon solar cells," Journal of Applied Physics, vol. 101, no. 2, 2007. 